\title{
Till Dembeck / Rolf Parr (Hrsg.): Literatur und Mehrsprachigkeit. Ein Handbuch
}

Till Dembeck, Rolf Parr (Herausgeber), Tübingen: Narr Francke Attempto 2017. ISBN 978-3-8233-7911-9. $380 \mathrm{~S}$.

\section{Martina KOFER ${ }^{10}$}

Universität Paderborn, Fakultät für Kulturwissenschaften/Institut für Germanistik und vergleichende Literaturwissenschaft, Paderborn

\section{Corresponding author:}

Martina KOFER, Universität Paderborn, Fakultät für Kulturwissenschaften/Institut für Germanistik und vergleichende Literaturwissenschaft, Warburger Str. 100, 33098 Paderborn, Deutschland

E-mail: martina.kofer@upb.de

Wie Unterricht und Schule mehrsprachig gestaltet werden können und die Mehrsprachigkeit der Kinder wertschätzend integriert werden kann, ist heute in vielen Einwanderungsländern zentrales Thema der Wissenschaften. Dass wir jedoch noch weit davon entfernt sind, Mehrsprachigkeit als alltäglichen Normalfall zu begreifen und als gesellschaftlich bereichernd anzuerkennen, zeigen beispielsweise Forderungen nach einem ,Verbot' anderer Sprachen als Deutsch auf dem Schulgelände. Yasemin Yıldız (2012) sieht in dieser auch institutionell geforderten ,Leitsprachigkeit' ein monolinguales Paradigma, das mehr ist, als das simple Sprechen einer Sprache. Vielmehr sieht sie in Einsprachigkeit ein strukturelles Prinzip zur Organisation des gesamten gesellschaftlichen Lebens. Auch im literarischen Feld 
zeigt sich eine merkwürdige Ambivalenz zwischen den lebensweltlichen multilingual geprägten Realitäten und der konsequenten Einsprachigkeitsdominanz in den ,Nationalliteraturen', wie David Gramling im vorliegenden Band konstatiert.

In den letzten Jahren tastet sich die Wissenschaft jedoch behutsam aber stetig an Mehrsprachigkeit als narratologisches Verfahren heran. Giulia Radaelli legte 2011 mit ihrer Dissertationsschrift eine erste Systematik zur Beschreibung literarischer Mehrsprachigkeitsverfahren vor, nachdem für lange Zeit auf diesem Feld nur die Aufsätze von András Horn (1981) und Meir Sternberg (1981) sowie das frühe Buch von Leonard Forster (1970) Orientierung boten. Die bisher umfangreichste Untersuchung einer "Ästhetik der Mehrsprachigkeit" (2016) legte jüngst der Romanist Werner Helmich vor. Während Helmich jedoch literarische Mehrsprachigkeit auf das Sprechen von mindestens zwei differenten Idiomen im Text reduziert und als literarisches Ausnahmephänomen betrachtet, vertreten Till Dembeck und Rolf Parr im nun vorliegenden Band eine Position, die die Allgegenwärtigkeit von Mehrsprachigkeit im Sozialen wie auch in literarischen Texten hervorhebt. Sie betrachten Mehrsprachigkeitsforschung als "neue disziplinäre Ausrichtung der Literaturwissenschaft" (S. 10).

Das anvisierte Ziel einer Mehrsprachigkeitsphilologie wird im Laufe der Lektüre des fast 400 Seiten umfassenden Handbuchs mit Blick auf die ästhetische Vielfalt immer ersichtlicher. So rechnen die Herausgeber nicht nur den Sprachwechsel bzw. die Sprachmischung verschiedener "Fremdsprachen' in der Literatur zur Mehrsprachigkeitspoetik. Der Polyphonie-Theorie Michail Bachtins folgend, zählen sie auch Aspekte innerer Mehrsprachigkeit zu den ästhetischen Erscheinungsformen. Zu den „Basisverfahren“ gehört jedoch auch, wie Till Dembeck im dritten Teil des Buches ausführlich darstellt, die Integration von metrischen Mustern oder Zitaten aus einem ,fremdsprachlichen' Kontext. Auch wenn eine, andere' Sprache nicht offensichtlich im Text präsent ist, können noch Verfahren von Mehrsprachigkeit analysiert werden, die oftmals nur von einem dementsprechend sprachkompetenten Rezipienten als immanente, wortwörtliche Übersetzungen erkannt werden. Selbst die bloße Erwähnung des Sprechens einer anderen Sprache im Text gehört als Form latenter Mehrsprachigkeit zum formellen Ensemble.

Ganz im Sinne der aktuellen Debatte um eine neue Weltliteratur', die die bisherigen nationalphilologischen Ordnungen von Literatur für überholt hält, sehen die 
Herausgeber in der literarischen Mehrsprachigkeitsforschung das Potenzial, die nationalphilologischen Eingrenzungen der Literaturwissenschaft zu durchbrechen. Aus ihrer Sicht erweitert Mehrsprachigkeit den Begriff der Weltliteratur, indem globale Vernetzung nicht nur auf die Texte und ihre Intertextualität bezogen wird, sondern sich auch in einem Netzwerk vielfältiger mehrsprachiger Poetiken zeigt. Trotz der ,Leitlinie Einsprachigkeit' ist Mehrsprachigkeit in der Literatur jedoch ebenso wenig wie in der sozialen Realität ein Globalisierungsphänomen, sondern ein hoch dynamisches ästhetisches und auch kulturpolitisches Prinzip der Weltliteraturgeschichte, das sich schon in antiken Texten findet und sich fortan in verschiedenen Formationen und wechselnden Sprachpräferenzen in jeder Epoche zeigt.

Mehrsprachige Literatur steht darüber hinaus immer im Kontext von Inter- und Transkulturalität und bietet von daher ein wichtiges Analysemoment von DifferenzKonstrukten - kultureller wie auch sozialer Art. So ist Mehrsprachigkeit den Herausgebern zufolge untrennbar mit (kultur-)politischen Dominanzverhältnissen und Hierarchisierungen verbunden, denn der Diskurs um Einsprachigkeit und Mehrsprachigkeit verhandelt immer schon die Themen kulturelle (Des-)Integration, ,Eigenes'/,Fremdes' im kulturellen wie im sozialen Sinne mit und hat von daher einen implizit ethischen Charakter. Wie sehr Sprache mit Machtverhältnissen verbunden ist und rassistischen Strukturen förderlich sein kann, hat nicht zuletzt die Linguistik selbst in ihrer Hierarchisierung von Sprachen im Rahmen der Kolonialpolitik vorgeführt. So betont David Gramling, dass die Kritik an der Sprache der ,Anderen' kein zwingend modernes Phänomen ist. Schon die Urerzählung Schibboleth im Alten Testament zeigt, wie Sprachigkeit in kriegerischen Auseinandersetzungen zum Entscheidungskriterium für, richtige' oder,falsche' Zugehörigkeit werden kann.

Die "kulturellen und sozialen Rahmenbedingungen literarischer Mehrsprachigkeit" werden insbesondere im ersten Teil des Bandes fokussiert. In Beiträgen von Till Dembeck wird zunächst auf das reziproke Verhältnis von Sprache und Kultur und seine identitären Auswirkungen eingegangen. Dembeck fokussiert die Ausbildung der Muttersprachensemantik zum Ende des 18. Jahrhunderts als Geburtsstunde der Einsprachigkeits- und Mehrsprachigkeitssemantik und ihrer kulturellen Auswirkungen bis in die Gegenwart. Er erinnert daran, dass mit der Wahrnehmung bzw. Herstellung von Sprachdifferenzen neben der Wertschätzung kultureller und sprachlicher Besonderheiten im 18. und 19. Jahrhundert auch Unsicherheiten kultureller wie sprachlicher Zugehörigkeit entstanden, die u.a. zu 
einer dominanten okzidentalen Identitätspolitik führten, die sich spätestens im Kolonialismus in ihrer ganzen Tragweite zeigte. Dembeck plädiert vor diesem Hintergrund für eine doppelte Perspektive auf Kultur in der Mehrsprachigkeitsforschung: Zum einen müssten Sprachdifferenzen im Sinne von Kulturdifferenzen als Analyseinstrument "gesellschaftlicher und anderer Strukturen und Prozesse" (S. 24) begriffen werden. Zum anderen seien Sprachdifferenzen aber immer auch kulturpolitisch interpretierbar, indem sie für Auseinandersetzungen über Bedeutungszuschreibungen stehen können und von daher „insbesondere die (kultur-)politische >Agency< von Literatur beschreibbar" (ebd.) werden lassen.

David Gramling sieht eine historische und systematische Analyse von Ein- und/ oder Mehrsprachigkeitsanschauungen "vor dem Hintergrund des jeweiligen sozialen, politischen und kulturellen Rahmens unabdingbar" (S. 39). Es geht ihm von daher bei der Bestimmung von Einsprachigkeit auch vorrangig um das ,Wie' des Werdens von einheitlichem Sprechen, den entsprechenden Zeitpunkten und der Rolle des Mediums Literatur zur Konstitution von Einsprachigkeit.

Jörg Roche untersucht die Bedeutung von Sprache im Kontext von Integration bzw. Desintegration und setzt sich mit der Situation von MigrantInnen auseinander. Unter den Bedingungen einer "politisch gewollten ,Leitsprachigkeit" (S. 45) sieht Roche "[d]ie Frage, wie genau welche Sprache(n) wo und wann Medium der Integration oder eben Desintegration sind, als einen immensen Einflussfaktor für "das Verständnis von mehrsprachiger Literatur" (S. 45) und schreibt dieser eine integrative Wirkung zu. Ethnolekte wie das Kiezdeutsch unterlaufen ihm zufolge leitsprachige Integrationsprämissen und entwickeln eine Eigendynamik, die sich auch in künstlerischen Formaten wie der Literatur, dem Kabarett etc. sprachlich transformieren.

Arvi Sepp geht der "Ethik der Mehrsprachigkeit" auf den Grund. Er sieht die Funktion mehrsprachiger Literatur in ihrem symbolischen Ausdruck der Varietät und der "Vermischung von Kulturen und Sprachen" (S. 53). Darauf basierend, dass sprachliche Kommunikation nicht $\mathrm{zu}$ trennen ist von Macht- und Herrschaftsverhältnissen, versteht er unter einer Ethik der Mehrsprachigkeit eine Bevorzugung von kommunikativen Handlungen, die einen Perspektivwechsel ermöglichen und eine Gleichberechtigung der Kommunizierenden verfolgen. Die für mehrsprachige Literatur typische Sprachreflexivität hat für inn "eine ethische 
Dimension", als dass sie die Mehrdimensionalität des Denkens und des gesellschaftlichen Zusammenlebens verdeutlichen kann.

Den zweiten Teil des Bandes zu "sprachlichen Rahmenbedingungen von Mehrsprachigkeit" einleitend, gibt Heinz Sieburg einen Überblick zu den Voraussetzungen der Sprachstandardisierung und der linguistischen Begrifflichkeiten des Varietäten-Spektrums wie Dialekt, Soziolekt, Fachsprache etc. Im Vergleich zur Standardsprache ist die Nationalsprache ihm zufolge vor allem ideologisch formatiert, während die Literatursprache entweder als Synonym oder Antonym zur Standardsprache steht. Für eine Einordnung als Weltsprache sei vor allem die numerische Stärke und das ,Prestige' einer Sprache entscheidend. Ergänzend definiert Sieburg Kontaktsprachen wie Pidgins und Kreolsprachen und Plan- oder auch Welthilfssprachen als künstliche Sprachsysteme.

Wie Sprachstandards überhaupt entstehen, erläutert Helmut Glück. Dazu gehört die Benennung und Regulierung einer Sprache und ihre Abgrenzung von anderen Sprachen ebenso wie eine ausgeprägte institutionelle Kontrolle, die über sprachliche Korrektheit und Qualität entscheidet. Teil dessen ist die ständige Überarbeitung und Anpassung dieser Standards durch Grammatikographen.

Abschließend wird eine "Pragmatik der Mehrsprachigkeit" im Artikel von Jörg Roche und Gesine Leonore Schiewer verhandelt. Dabei sprechen sich beide grundsätzlich für eine stärkere Verbindung von Linguistik und literaturwissenschaftlicher Mehrsprachigkeitsforschung aus. Methodische Ansätze wie Sprechakttheorie und Gesprächsanalyse können in pragmalinguistischen Untersuchungen von Mehrsprachigkeit innen zufolge gewinnbringend sein. Die Interkulturelle Literatur mehrsprachiger Autorlnnen bietet zahlreiche Beispiele, wie die verschiedenen multilingualen Spielarten im Text unterschiedlich linguistisch fundiert sein und Ebenen "von der phonetisch-phonologischen bis zu der von Texten und gegebenenfalls Diskursen“ (S. 116) einbeziehen können.

Ein Artikel zu Mehrschriftlichkeit in literarischen Texten von Monika Schmitz-Emans ergänzt die Beiträge von Till Dembeck im dritten Teil des Bandes. Entscheidend für die Untersuchung ist hier vor allem die Reichweite des Schrift-Verständnisses. Dabei wird ihr zufolge in der Gegenwartsliteratur "Mehr- und Vielschriftlichkeit in ihren verschiedenen Dimensionen" (S. 230) zunehmend ästhetisch positioniert, was sich 
mit dem Interesse am kulturell Besonderem wie Differenten erklären lässt. Nicht nur die steigende Beliebtheit von Graphic Novels als literarisches Genre, auch die zunehmende Präsenz graphischer Darstellungen im literarischen Text motiviert dabei eine intensivere Beschäftigung mit Mehrschriftlichkeit in der Gegenwartsliteratur.

Dem wichtigen Thema der Übersetzung gilt der vierte Teil des Bandes. Hier wird unterschieden zwischen den beiden Verfahren semantische Übersetzung (Henri Bloemen und Arvi Sepp) und homophone Übersetzung (Till Dembeck). Bezogen auf Derrida vertreten Bloemen und Sepp ein Verständnis von Übersetzung als ein "unabschließbarer semiotischer Prozess der Zeichen- und Bedeutungsinterpretation, die stets ideologisch und kulturell bedingt ist" (S. 241). Der damit einhergehende sprachreflexive und kulturkritische Charakter der Übersetzung betrifft den Autoren zufolge vor allem auch die mehrsprachige Literatur, da dieser die Auseinandersetzung mit sprachlichen und kulturellen Dichotomien immer schon immanent ist. Eine wissenschaftliche Auseinandersetzung mit (literarischer) Mehrsprachigkeit ginge von daher immer auch einher mit übersetzungstheoretischen Überlegungen.

Till Dembeck erkennt in der Anwendung homophoner Übersetzungen vor allem ihre Tendenzhaftigkeit: "die Art und Weise, wie der Originaltext im Übersetzungsvorgang womöglich strategisch auf eine bestimmte Bedeutung hin ,abgehört' wird" (S. 249). Als wichtige Aufgaben in der noch sehr jungen Forschung sieht Dembeck "die Erfassung des historischen Materials" und "die Entwicklung eines analytischen Instrumentariums zur Beschreibung der intrinsischen Vielfalt homophoner Übersetzungen“ (S. 255).

Das letzte inhaltliche Kapitel (V.) beleuchtet literarische Mehrsprachigkeit aus gattungs- und medienspezifischer Perspektive. Till Dembeck zentriert in seinem Beitrag zur Versform jene Verfahren, die metrische Komponenten aus differenten sprachlichen und kulturellen Bezugsräumen miteinander mischen. Dabei legt er sowohl die Beziehung zwischen der Geschichte des mehrsprachigen Versbaus und seiner sprachgeschichtlichen Entwicklung dar, als auch deren bedeutenden Einfluss auf die Entwicklung „der (neuzeitlichen) Lyrik als Gattungszusammenhang“ (S. 260).

Im Theater werden Sprachvarietäten besonders gerne eingesetzt, um Figuren entsprechend zu charakterisieren und sozial zu verorten. Dabei geht mit dem Einsatz verschiedener figurenspezifischer Sprachvarietäten oftmals auch - wie Claude D. 
Conter in seinem Beitrag zur Dramatik darlegt - eine sozialkritische Intention des Autors / der Autorin einher. Diese kann auch „Instrument sozialpolitisch motivierter Ideologiekritik" (S. 278) sein. An Figurentypen wie des Gelehrten oder des Fremden offenbart sich eine "genrespezifische [...] Funktion manifester Mehrsprachigkeit" im Theater. Entgegen der marginalen Forschung zu mehrsprachiger Dramatik im europäischen Raum ist im Zuge des Postkolonialismus durchaus eine Forschung zu Mehrsprachigkeit und Theater in den ehemaligen Kolonien festzustellen. So kann Mehrsprachigkeit z.B. "als dramaturgische Strategie von Ausgrenzung bzw. Einbeziehung verschiedener ethnischer Gruppen“" (S. 285) Verwendung finden.

Rüdiger Zymner konstatiert in der Forschung zu Mehrsprachigkeit und „Erzählen“ eine Leerstelle. So wird ihm zufolge Mehrsprachigkeit in Texten nur selten in narratologischer Sicht interpretiert. Stattdessen dominiere ein literaturhistorischer und geistesgeschichtlicher Fokus die Interpretationen. Als notwendig für die weitere Forschung betrachtet er eine breitgefächerte Systematik, die alle „'Bauelemente' der Erzählung/des Erzähltextes" (S. 296) - erzählender wie außererzählender Rede, Paratexte etc. - mitberücksichtigt.

Die populärste und wohl anerkannteste künstlerische Form von Mehrsprachigkeit ist wohl die des Liedtextes, wie Anne Uhrmacher in ihrem Beitrag überzeugend zeigt. Hier sind die Möglichkeiten multilingualer Sprachspiele nahezu unbegrenzt. Hervorzuheben ist die enge Beziehung der Popularmusik zu verschiedenen Jugendkulturen und die damit einhergehenden Besonderheiten in der Wahl der Sprachen. So herrschen vor allem englischsprachige Einschübe sowie soziolektale, dialektale und ethnolektale Sprechweisen vor. Nicht empirisch überprüfbar ist hingegen "wie Liedtexte früherer Jahrhunderte zur Abgrenzung gesellschaftlicher Gruppen beitragen konnten" (S. 301). Gesellschaftliche Abgrenzung wird heute ebenso wie Subversion immer wieder in den Texten thematisiert. Das Spiel mit Sprachdifferenz kann daneben, wie die Autorin hervorhebt, nicht nur Sprachskepsis erzeugen, sondern auch als Identitätsmarker eingesetzt werden.

Das Hörspiel und Hörbuch besitzt das Potenzial, in Form von fremdsprachiger Aufnahmen zum Beispiel anderssprachliche Zitate als "stilistische Markierungen bestimmter Sprechweisen oder Status- und Milieumarkierungen“ (S. 309) gezielt herstellen zu können. Diese machen - wie Natalie Binczek betont - Mehrsprachigkeit hörbar. Besonders bei Formen innerer Mehrsprachigkeit wie Dialekten kann ein 
spezifisch artikulatorischer Akzent (vgl. S. 310) hervorgehoben und als dramaturgisches Mittel eingesetzt werden. Die spezifische Ebene der akustischen Umsetzung des Hörbuchs und Hörspiels erwirkt, "dass Effekte der Fremd- und Mehrsprachigkeit sich hier auch als ein Hybrid aus Lexik und Syntax einer Sprache einerseits und dem artikulatorischen Akzent einer Sprache andererseits hervorbringen lassen" (S. 312f).

Zwei Aufsätze zu Film (Claude Kremer) und Fernsehen (Rolf Parr) vervollständigen den genrespezifischen Blick auf Mehrsprachigkeitsverfahren. Claude Kremer folgend, ist es der Globalisierung und der dynamischen Entwicklung von Genrekonventionen zu verdanken, dass ein weniger stereotyper Sprachgebrauch in den Filmen zu bemerken ist. Durch die im Vergleich zur Literatur fehlende Erzählerinstanz, müssen die filmischen Mittel zur Charakterisierung einer Figur in Hinblick auf eine Sprachgemeinschaft viel genauer ausgelotet werden. Die akustische Umsetzung ermöglicht auch hier, dass die Zuschauer und Zuhörer direkt mit dem Klang der Sprache konfrontiert werden und dadurch eine eigenständige Entzifferung fremder Zeichen wegfällt. Dies befördert nach Kremer ein erweitertes Repertoire an mehrsprachigen Elementen im Film. Rolf Parr setzt sich in seinem Beitrag zum "Fernsehen" mit "Iiteraturnahen' fiktionalen Fernsehnarrationen" (S. 329) auseinander, die Aspekte integrativer Mehrsprachigkeit aufweisen. Die besondere Herausforderung für das Fernsehen besteht dabei darin, Formen manifester und latenter Mehrsprachigkeit so zu inszenieren, dass auch den Sprachen nicht mächtige Zuschauerlnnen dem Inhalt der Sendungen folgen können. Parr erläutert zwölf verschiedene Verfahren und favorisiert letztlich die „in der eigenen Sprache simulierte Mehrsprachigkeit" (S. 331). Gerade in der Integration fremdsprachlicher Grammatikstrukturen in die Basissprache sieht Parr die Möglichkeit, "Mehrsprachigkeit integral [zu] realisieren" (S. 332). Darin sieht er eine Parallele zu Homi K. Bhabhas Theorie des Dritten Raums, in dem durch das Verschmelzen von Identität und Differenz neue hybride Formen entstehen. Dieser Raum der simulierten Mehrsprachigkeit ist jedoch im Gegensatz zu Bhabhas ,drittem Raum' "gerade nicht hierarchiefrei" (S. 332) - die Dominanz der Basissprache bleibt bestehen - trotz evtl. zusätzlicher fremdsprachlicher Einsprengsel in die Figurenrede. Im engen Verhältnis der Verfahren der Sprachvermengung und -simulation zu den Figuren(-gruppen) und der semantisierten Räume sieht Parr das Potenzial, „Effekte von multikultureller Authentizität zu erzielen, die v.a. aus Kollisionen sprachlicher, kultureller und zugleich räumlicher Art resultieren" (S. 335f). Insgesamt entstehe so ein „fluide[r] transkulturelle[r] Raum, in Bewegung' [...], 
der sich seinerseits aus einer Vielzahl kleinerer, meist selbst schon hybrider transethnischer Kulturen zusammensetzt" (S. 336).

Der von Dembeck und Parr gesetzte methodische Schwerpunkt und die damit einhergehende Erarbeitung eines Analyse-Instrumentariums der "strukturelle[n] Gefüge von Sprachdifferenzen im Text" (S. 10) und ihrer kulturpolitischen Bedeutungsebene, deckt einen dringenden Bedarfin der Mehrsprachigkeitsforschung ab und bietet zugleich eine optimale Basis für weiterführende Forschungen.

Zu bemängeln ist, vor allem im Kontext des hergestellten Bezugs zur WeltliteraturDebatte, die einseitige Orientierung an europäischen Literaturen. Die Verfahren der Mehrsprachigkeit können m.E. erst in ihrer ganzen Bandbreite und vielfältigen ästhetischen Kreativität erfasst werden, wenn auch Texte afrikanischer, asiatischer u.a. Literaturen und ihrer Sprachen miteinbezogen werden. Zugute zu halten ist den Herausgebern hier, dass ihnen diese Lücke durchaus bewusst ist (vgl. S. 144). Die Erforschung von Mehrsprachigkeit in globaler Dimension bringt darüber hinaus ein grundlegendes methodisches Problem mit sich: Wie mehrfach im Band erwähnt, stellt mehrsprachige Literatur gerade auch an den Rezipienten bzw. den Forschenden hohe (mehrsprachige) Anforderungen. Eine intensivere interdisziplinäre Zusammenarbeit (S. 162) bzw. die Arbeit in transkulturellen multilingualen Forschungsteams (S. 119) ist von daher eine dringende Voraussetzung für eine umfassende Forschung. Hier sind vor allem auch mehrsprachig aufgewachsene Wissenschaftlerlnnen gefragt, die leider auch heute noch nur ein fremdsprachiges Einsprengsel in der nationalphilologisch definierten Literaturwissenschaft ausmachen.

\section{Literaturverzeichnis}

Forster, Leonard: The Poet's Tongues. Multilingualism in Literature. The de Carle Lectures at the University of Otago 1968. Cambridge: University Press 1970.

Helmich, Werner: Ästhetik der Mehrsprachigkeit. Zum Sprachwechsel in der neueren romanischen und deutschen Literatur. Heidelberg: Winter 2016.

Horn, András: Ästhetische Funktionen der Mehrsprachigkeit in der Literatur. In: arcadia 16 (1981), S. 225-241.

Radaelli, Giulia: Literarische Mehrsprachigkeit. Sprachwechsel bei Elias Canetti und Ingeborg Bachmann. Berlin: Akademie Verlag 2011.

Sternberg, Meir: Polylingualism as Reality and Translation as Mimesis. In: Poetics Today 2/4 (1981), S. 221-239.

Yıldız, Yasemin: Beyond the Mother Tongue. The Postmonolingual Condition. New York: Fordham University Press 2012. 
